\title{
Screening of inherited metabolic abnormalities in 56 children with intractable epilepsy
}

\author{
XIAOMING LIU, RUI LI, SHENGZHI CHEN, YAN SANG and JIAQIANG ZHAO \\ Department of Neurology, Xuzhou Children's Hospital, Xuzhou, Jiangsu 221002, P.R. China
}

Received November 19, 2015; Accepted April 14, 2016

DOI: $10.3892 /$ etm.2016.3260

\begin{abstract}
Epilepsy is a common children's neural disease that is largely controlled by anti-epileptic drugs. Nevertheless, children experience repeated attacks that develop into intractable epilepsy (IE). The aim of the present study was to examine the inherited metabolic abnormalities in children with IE to provide early etiological and symptomatic treatment. Urine and blood samples of 56 children with IE served as the experimental group and 56 cases of children with IE, who were successfully treated served as the control group, and analyzed by gas chromatography-mass spectrometry and tandem mass spectrometry for the metabolic screening of amino, organic, and fatty acids. Urine routine, hepatic function, blood biochemistry, imageology of encephalon and brain stem-evoked potential (auditory and optical) were also examined. Of the 27 IE children confirmed as abnormal in urine and blood screening, there were 19 cases $(70.3 \%$ ) of hypoevolutism or retrogression of intelligence and motor function, 15 cases $(55.5 \%)$ of brain stem-evoked potential and of encephalic computed tomography (CT) or magnetic resonance imaging (MRI) abnormality, 6 cases (22.2\%) of abnormal family history and of abnormal blood biochemistry and blood gas analysis, and 5 cases $(18.5 \%)$ with skin change and of abnormal hepatic function. Of the 27 cases, 11 cases $(19.6 \%)$ were diagnosed with inherited metabolic diseases. Among the children in the control group, 3 cases showed abnormal urine test results, one of which had family history, one had hypoevolutism or retrogression of intelligence and motor function, one had brain stem-evoked potential and encephalic CT or MRI abnormality, while two of the 3 cases had inherited metabolic abnormalities. The correlation analysis revealed that abnormal urine test was significantly correlated with inherited metabolic abnormalities $(\mathrm{P}<0.05)$. Of the 56 IE patients, 25 cases $(44.6 \%)$ were identified as abnormal under urine screening, and of the 25 cases,
\end{abstract}

Correspondence to: Dr Rui Li, Department of Neurology, Xuzhou Children's Hospital, 18 Sudibei Road, Xuzhou, Jiangsu 221002, P.R. China

E-mail: xzlrlxm@163.com

Key words: intractable epilepsy, inherited metabolic abnormality, spasm
6 cases had simple generalized seizure (24.0\%), 5 cases had simple partial seizure $(20.0 \%), 2$ cases had multiple types of seizure $(8.0 \%)$, and 12 cases had infantile spasms $(48.0 \%)$. Six cases in the control group showed an abnormal urine test, with 3 cases of simple generalized seizure, 2 cases of simple partial seizure and 1 case of multiple types of seizure. The abnormal urine in infantile spasms was often the risk factor of IE. A total of 46 IE children underwent blood screening and 13 cases were diagnosed as abnormal. In conclusion, inherited metabolic abnormality was a common influential factor in the pathogenesis of IE, especially in infantile spasms. Screening of inborn metabolic abnormality in children with IE should be conducted as early as possible, to achieve early treatment and improve their prognosis.

\section{Introduction}

Epilepsy is a common children's neural disease (1). Most epileptic seizures are controlled by anti-epileptic drugs $(2,3)$. Nevertheless, 20-30\% of children experience repeated attacks that develop into intractable epilepsy (IE) $(4,5)$. The pathogenesis of IE is complicated, and remains to be clarified. The development of molecular genetics and biochemistry have led to the finding that IE is closely associated with inherited metabolism. IE has always been a problematic issue in neurology, and a hot spot of current researches. Particularly in the infantile period when the brain of children develops rapidly, IE was more liable to cause different degrees of injury on the neuropsychological development of children $(6,7)$. At present, studies on IE pathogenesis are mainly focused on hereditary metabolic disorders and gene expression abnormalities, neural network structural and functional abnormalities and multidrug resistance formation (8). An increasing number of children with IE have been confirmed to be associated with heredity. Inherited metabolic diseases (IMDs) $(9,10)$ are a series of metabolic defects of different clinical manifestations, including organic acid, amino acid, and fatty acid metabolic diseases, which are incurred by disorders of biochemical metabolism, and the accumulation or insufficiency of metabolites resulting from the structural defects or functional disorders of protein arising from gene mutation (11-13).

Since most IMDs are lacking in specific symptoms, they are easily ignored or missed in clinical diagnosis, resulting in serious psychomotor development disorders, and even death. Although IMD was lacking in specific symptoms, nerve 
systems were highly involved, which manifested in convulsion attacks and developmental retardation. Therefore, epileptic children, especially children with IE should be examined to exclude this type of disease. In the present study, we screened inborn metabolic abnormality in children with agnogenic IE.

\section{Materials and methods}

Study objects. A total of 56 children with IE were enrolled between June 2009 and August 2012. The children were outpatients or inpatients at the Xuzhou Children's Hospital (Jiangsu, China) and conformed to the unified criteria of IE as defined by the Special Working Group of International League Against Epilepsy. The unified criteria for the present study were: Clinical diagnosis was exact, the application of two types of appropriate and tolerable antiepileptic drugs failed to completely preven epileptic seizure follownig adequate duration of treatment (duration free from any epileptic seizure was $\geq 3$-fold the longest interictal period prior to treatment or was 12 months) and adequate doses of single or combined drugs, or patients were clinically confirmed as IE and having epilepsy syndrome. Since the longest interictal periods in the enrolled patients were $<3$ months, 12 months were defined as the course of treatment. Intracranial mass, head trauma, perinatal cerebral injury, poisoning, injection, and other clear etiologies were excluded. Of the 56 cases, there were 14 cases of generalized seizure, 16 cases of partial seizure, 19 cases of infant spasms, and 7 cases of combined generalized and partial seizure. There were 37 male and 19 female patients, with an average age of 2.1 years. Specimens of the 19 children experiencing infant spasm were collected following diagnosis and before the application of antiepileptic drugs. Other cases were under the treatment of antiepileptic drugs, free from pneumonia, dysentery, or any other complications. In the control group, there were 12 cases of generalized seizure, 17 cases of partial seizure, 18 cases of infant spasms and 9 cases of combined generalized and partial seizure. There were 36 male and 20 female subjects, with an average of 2.2 years. The data of the two groups were compared and the difference identified was not statistically significant $(\mathrm{P}>0.05)$.

Urine screening. A specialized urine strip (Shenzhen Huiyan Kechuang Biological Technology Co. Ltd., Shenzhen, China) was used to store the urine specimen, urease to conduct pretreatment, and gas chromatography-mass spectrometry (GC/MS) to analyze the components of the urinary metabolite. The Japan Electron Optics Laboratory (JEOL) GC/MS analyzer (Jms-Q1000GC; JEOL Ltd., Kyoto, Japan) was used and the electron impact method was applied to produce MS.

Blood screening. Three drops of peripheral blood were collected and dripped on specialized filter paper. The blood was left under normal temperature $\left(10-30^{\circ} \mathrm{C}\right)$ for natural drying, and tandem mass spectrometry (MS/MS) was applied to analyze the blood metabolite.

Other examinations. The enrolled children underwent a series of examinations, including blood sugar examination, blood electrolytes examination, hepatorenal function examination, urine routine examination, electroencephalogram, brainstem-evoked response (auditory and optical), and skull imaging. Pediatric internal medicine examination and nervous system examination were conducted routinely. Certain cases underwent biochemical examinations on blood ammonia, blood gas analysis, and blood lactic acid.

\section{Results}

Screening result. A total of 56 IE children underwent urine screening. The result showed that 31 cases were normal while the remaining 25 cases had the possibility of inborn errors of metabolism, of which 12 cases (48\%) had infant spasms, and was the most common type (Table I). Ten cases did not undergo blood screening due to family financial constraints, whereas the remaining 46 cases underwent blood screening. Of the 46 cases, 5 cases had increasing propionyl carnitine/acetyl carnitine $(\mathrm{C} 3 / \mathrm{C} 2)$ and propionyl carnitine; 3 cases had increasing phenylalanine; 1 case had glutaryl carnitine (C5DC) and decoyl carnitine (C5DC/C8); 1 case had citrulline; 1 case had a mild increase of 3-hydroxy 16 acylcarnitine $(\mathrm{C16OH}), 3$-hydroxy 18 acylcarnitine $(\mathrm{C18OH})$ and 3-hydroxy 18 acylcarnitine:1 (C18:1-OH); 1 case had a mild increase of non-specific abnormality, including arginine, glycine (Gly), and decanedioyl carnitine (C10DC); and 1 case had a mild increase of leucine (Leu)/isoleucine and methionine. According to the typical changes of urine and blood screening and their clinical performances as well as other examinations, 11 cases were confirmed with IMD, with a total positive rate of $19.6 \%$. Of the 11 confirmed cases, there were 5 cases of methylmalonic acidemia, 3 cases of phenylketonuria, 1 case of type II citrullinemia, 1 case of type I glutaric aciduria, and 1 case of 4-hydroxybutyrate acidemia (Table II).

Clinical performances of the children confirmed with abnormalities in screening as well as other examinations. Of the 27 cases of children with IE that were diagnosed with abnormalities in urine or blood screening, there were 19 cases $(70.3 \%)$ confirmed with backward or regressive intelligent movement; 15 cases $(55.5 \%)$ with skull imaging abnormalities, including cortex, alba, or callosum maldevelopment, hydrocephalus, and tricorn asymmetry; 15 cases $(55.5 \%)$ with brainstem-evoked response abnormalities; 5 cases (18.5\%) with liver function abnormalities (increase of transaminase) (27); 6 cases $(22.2 \%)$ with blood biochemistry and blood gas analysis abnormalities, including the increase of blood ammonia, and blood lactic acid; and 5 cases $(18.5 \%)$ with skin change, 2 cases of which had phenylketonuria, mainly manifesting in the reduction of skin pigment; 1 case had lactaciduria, manifesting as hyperpigmentation; 1 case had ketotic dicarboxylic aciduria, manifesting as pachulosis and skin peeling; and 1 case with tryptamine hydrochloride aciduria, manifesting as photosensitive dermatitis (Table III).

A survey of family medical history. Of the cases confirmed with abnormalities in urine or blood screening, 6 cases (22.2\%) had a family medical history. Of the 6 cases, there were 2 cases where one parent had a history of epilepsy (1 case of non-ketotic dicarboxylic aciduria and 1 case of lactaciduria); 1 case whose father had amentia (ketotic dicarboxylic aciduria); 1 case whose mother had amentia, accompanied 
Table I. Seizure types of the 24 children with abnormalities in urine screening.

Seizure types

Urine screening abnormality

\begin{tabular}{|c|c|c|c|}
\hline \multicolumn{4}{|c|}{ Seizure types } \\
\hline General & Partial & General + partial & Infantile spasm \\
\hline
\end{tabular}

Ketotic dicarboxylic aciduria, 5 cases

Methylmalonic aciduria, 5 cases

Non-ketotic dicarboxyl aciduria, 3 cases

Phenylketonuria, 3 cases

Lactaciduria, 1 case

Glutaric aciduria, 1 case

2

2

2
0

0

1

0

0

Propionic aciduria, 1 case

$0 \quad 0$

4-Hydroxypropiophenone aciduria, 1 case

$0 \quad 0$

4-Hydroxybutyl aciduria, 1 case

Lactose and galactose metabolic abnormality, 2 cases

$1 \quad 0$

02

Pyroglutamic aciduria, 1 case

$0 \quad 0$

Tryptamine hydrochloride aciduria, 1 case

$0 \quad 0$

Total: 25 cases

6

$\begin{array}{rrr}2 & 0 & 1 \\ 0 & 1 & 2 \\ 1 & 1 & 1 \\ 0 & 0 & 2 \\ 0 & 0 & 1 \\ 0 & 0 & 1 \\ 0 & 0 & 1 \\ 0 & 0 & 1 \\ 0 & 0 & 0 \\ 2 & 0 & 0 \\ 0 & 0 & 1 \\ 0 & 0 & 1 \\ 5 & 2 & 12\end{array}$

Table II. Comparisons of the screening results of urine and blood on 56 IE patients.

\begin{tabular}{|c|c|c|}
\hline Blood screening & Urine screening & Clinical diagnosis \\
\hline Phe $\uparrow, 3$ cases & Phenylketonuria, 3 cases & Phenylketonuria, 3 cases \\
\hline $\mathrm{C} 3 \uparrow \mathrm{C} 3 / \mathrm{C} 2 \uparrow, 5$ cases & Methylmalonic acidemia, 5 cases & Methylmalonic acidemia, 5 cases \\
\hline $\mathrm{C} 5 \mathrm{DC} \uparrow \mathrm{C} 5 \mathrm{DC} / \mathrm{C} 8 \uparrow, 1$ case & Glutaric aciduria, 1 case & Type I glutaric aciduria, 1 case \\
\hline $\mathrm{C} 16 \mathrm{OH}, \mathrm{C} 18 \mathrm{OH}, \mathrm{C} 18: 1-\mathrm{OH}$ mild $\uparrow, 1$ case & Normal, 1 case & $\begin{array}{l}\text { Suspicious tri-functional } \\
\text { hypoproteinosis }\end{array}$ \\
\hline $\operatorname{Arg} \uparrow \mathrm{Gly} \uparrow \mathrm{C} 10 \mathrm{DC}$ mildly $\uparrow, 1$ case & $\begin{array}{c}\text { Ketotic double carboxylic acid } \\
\text { complicated by lactaciduria, } 1 \text { case }\end{array}$ & Further enzymatic analysis \\
\hline Leu-Ile, Met mildly $\uparrow, 1$ case & Normal, 1 case & - \\
\hline $\operatorname{Cit} \uparrow \uparrow, \operatorname{Met} \uparrow, 1$ case & Lactosuria and galactosuria, 1 case & Type II citrullinemia, 1 case \\
\hline Without abnormality, 33 cases & $\begin{array}{c}\text { 4-Hydroxybutyl aciduria, } 1 \text { case; } \\
\text { Ketotic double carboxyl aciduria, } 4 \text { cases; } \\
\text { lactaciduria, } 1 \text { case; non-ketotic } \\
\text { dicarboxylic aciduria, } 3 \text { cases; } \\
\text { lactose and galactosemetabolic } \\
\text { abnormality, } 1 \text { case; } \\
\text { 4-hydroxypropiophenone aciduria, } \\
1 \text { case; normal, } 22 \text { cases, }\end{array}$ & 4-Hydroxybutyl aciduria, 1 case \\
\hline Rejection, 10 cases & $\begin{array}{c}\text { Propionic aciduria, } 1 \text { case; } \\
\text { pyroglutamic aciduria, } 1 \text { case; } \\
\text { tryptamine hydrochloride } \\
\text { aciduria, } 1 \text { case; } \\
\text { normal, } 7 \text { cases }\end{array}$ & \\
\hline
\end{tabular}

Phe, phenylalanine; C3, propionyl-carnitine; C2, acetylcarnitine; C5DC, glutaryl acyl carnitine; C8, decoyl acyl carnitine; C16OH, 3 hydroxy palmitoylcarnitine; $\mathrm{C} 18 \mathrm{OH}, 3$ hydroxy stearylcarnitine; $\mathrm{C} 18: 1-\mathrm{OH}, .3$ hydroxy stearoyl-carnitine; C18:1-OH, 3 hydroxy stearylcarnitine:1; Arg, arginine; Gly, glycine; C10DC, decane-dioyl carnitine; Leu-Ile, leucine/isoleucine; Met, methionine; Cit, citrulline.

with deformed face (glutaric aciduria); and 2 cases with sibling deaths. The performance of the sibling of the case with non-ketotic dicarboxylic aciduria included acute onset, uncontrollable convulsion, emesis and dyspnea, while the sibling of the other case with citrullinemia was diagnosed with hemorrhagic disease of the newborn and abnormal liver function and succumbed to alimentary tract hemorrhage and intracranial hemorrhage at the age of eight. 


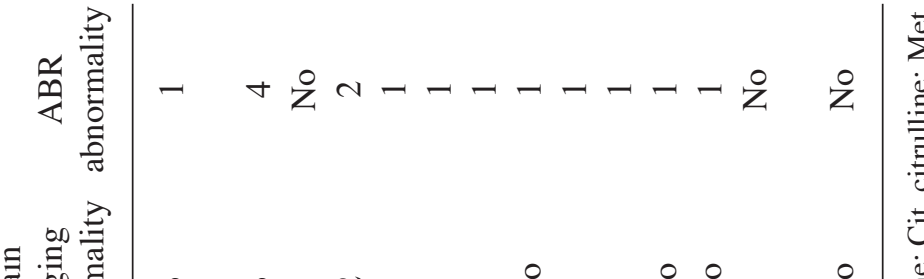



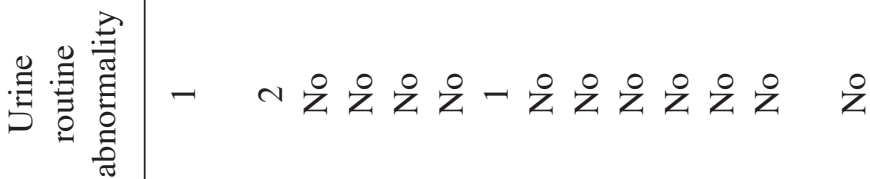

要

ต

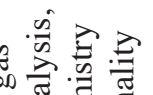

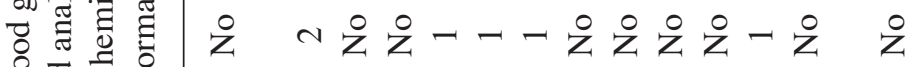

슬

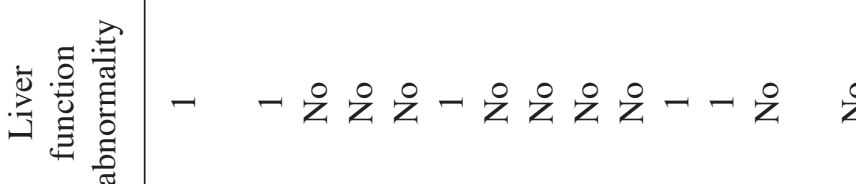

恶悬

过

艄.

突

离竞

焉

บิำ.

変

乙

을

需

死市

is

Uิ

苛

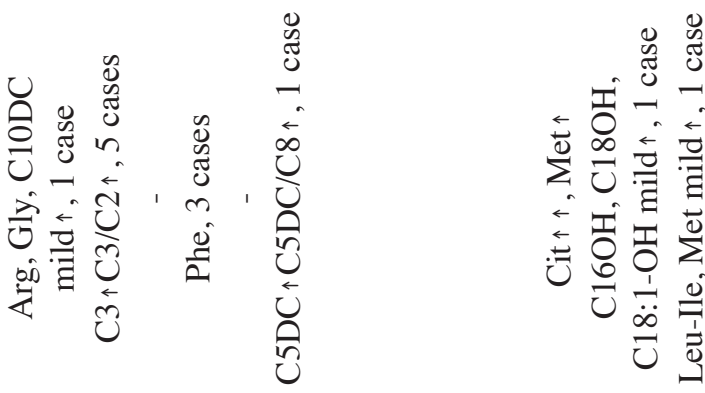

氖

言,

를

ชึ?

兽

奉㐫

-

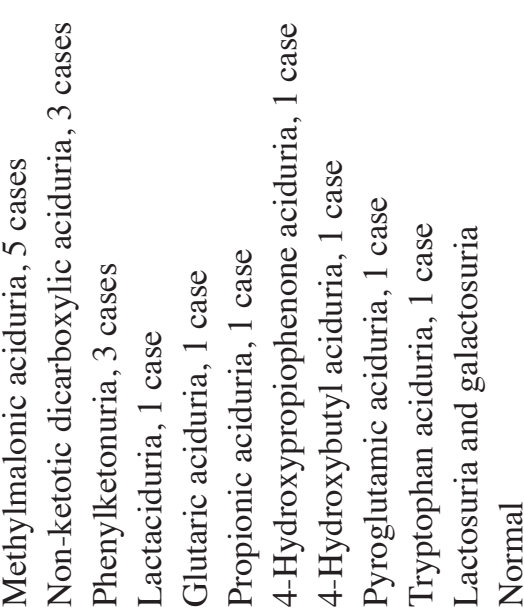


Therapeutic observation on confirmed cases. In addition to antiepileptic drug treatment, the 11 IE children diagnosed with IMD in the present study were administered a corresponding etiological treatment, symptomatic supportive treatment, and rehabilitation treatment, including diet therapy, a supplement of L-carnitine and vitamin, an application of aminocaproic acid, and a rectification of acidosis, and hyperammonemia. The duration of the follow-up period was 6 months. Of the 5 cases of methylmalonic acidemia, 1 case, due to failure in adhering to the recommended diet therapy and vitamin B12 treatment, became worsened in the control of epileptic seizure and development of motor and intelligence; 1 case, whose development of intelligent movement was diagnosed with significant backwardness following diagnosis at 2 years of age, had no significant improvement following treatment, but the control of their epilepsy had become ameliorated; and in the 3 cases of infant spasm, 2 cases had completely controlled epileptic seizure, while in the 1 case epileptic seizure had been reduced by $75 \%$ and intelligent movement was significantly improved, spirits and appetite were good and physical development was normal. The tetrahydrobiopteri metabolism of the 3 cases with phenylketonuria was confirmed as normal under urinary pterin analysis, with 2 cases aged 4 and 6 months and starting from infant spasm. After diet therapy and antilepileptic treatment, their epileptic seizure was completely controlled and intelligent movement greatly improved. The other case was lost to follow up. Epileptic seizure on 1 case with citrullinemia type II was effectively controlled, and the liver function and intelligent movement was returned to normal following the diet therapy, a supplement of lipid-soluble vitamin and treatment of blood ammonia reduction. Epileptic seizure on 1 case with glutaric aciduria type I was controlled, and was free from any acute seizure. Intelligent movement on 1 case with 4-hydroxybutyrate aciduria was greatly improved, and epileptic seizure was controlled following treatment, although the extrapyramidal symptoms had no significant improvement.

\section{Discussion}

The combination of MS/MS to detect the serum amino and fatty acid profiles, and GC/MS to detect urinary organic acid is widely used in clinical practice. It has provided exact diagnostic criteria for IDM diagnosis as well as a theoretical basis for its treatment (15-17). In the present study, we applied GC/MS to detect the urinary metabolic components of $56 \mathrm{IE}$ children, and 25 cases were confirmed with 12 types of abnormalities, of which there were 5 cases of methylmalonic aciduria, 5 cases of ketotic dicarboxylic aciduria, 3 cases of phenylketonuria, and 3 cases of non-ketotic dicarboxylic aciduria. In addition, we applied MS/MS to conduct blood screening on 46 IE children and 13 cases were identified as abnormal. Among the cases with abnormalities in urine and/ or blood screening, the metabolic components of 11 IE children had typical changes and were confirmed with IMD, with a positive rate of $19.6 \%$, and methylmalonic acidemia being the most common type.

Compared with the positive rate of the screened IMDs in clinical high-risk infants reported in literature worldwide (18), the positive rate of the group in the present study was relatively higher, which indicated that children with IE may have a higher incidence of IMD. However, our study included a limited number of samples, resulting in high selectivity of the cases, leading to deviation in the incidence of IMD. Thus, future studies should include more samples to verify the results of the present study. Except for the cases confirmed with IMD, other cases, which also showed metabolic disorders, should undergo further examinations with regard to enzymatic activity or relevant gene test (19-21). However, since conditions of hospitals in the Xuzhou region are lacking or limited, it has been suggested that child patients experiencing this disease, be sent to a high-level hospital for examination to confirm or exclude IMD. In one case in our group, which was diagnosed with lactaciduria in the initial urine screening and confirmed without significant lactic acid increase in the reexamination of urine screening two months later, a SCINA gene mutation, considered to be Dravet syndrome was identified.

In the 27 IE children that were confirmed as abnormal in urine and blood screening, $70.3 \%$ of cases were accompanied with developmental delay or regression of intelligence movement; $55.5 \%$ had head imaging and brainstem response abnormalities; and $18.5-22.2 \%$ had other abnormalities, including skin change, particular odor, liver function abnormalities, blood biochemical and blood gas analysis abnormalities as well as other family history abnormalities. Thus, agnogenic IE children should undergo relevant screening in the early period to exclude the possibility of IMD, especially when they exhibit the abovementioned performances. Compared with other forms of epileptic seizures, infant spasm was the most common type. In the present study, this result was obtained on 56 IE children, a limited number of patients. Thus, future studies should be conducted on more samples to verify the results obtained in the present study.

The catabolic pathways of antiepileptic drugs, such as valproic and fatty acid, may act together to affect organic acid, amino acid, and urea cycle metabolism, which if used over a long period of time, potentially lead to hyperammonemia $(22,23)$. Therefore, children with s suspicious urea cycle abnormality shoud undergo amino acid analysis prior to the use of valproic acid, the latter of which is to be used with caution. Long-term administration of valproic acid may affect the metabolism of branched chain amino acid, especially Leu and valine, as well as the $\beta$-oxidation inside mitochondria and reduce its function (24-27). Thus, close monitoring of the condition of valproic acid metabolite and branched chain amino acid is recommended.

In the present study, we provided corresponding etiological, symptomatic supportive, and rehabilitation treatments on the 11 IE children diagnosed with IMD and conducted a 6-month follow-up. Of these, one case was lost to follow-up and one case failed to adhere to the recommended diet therapy. The remaining 9 cases improved to varying degrees. The intelligent movement and epilepsy in younger children showed the most significant improvement. Thus, screening and diagnosis on younger IE children should be carried out as early as possible for early intervention and proper treatment to be provided, thereby effectively controlling patient seizures and improving their distant prognosis. To achieve this, large-scale studies should be performed. 


\section{References}

1. Durmus N, Kaya T, Gültürk S, Demir T, Parlak M and Altun A: The effects of $\mathrm{L}$ type calcium channels on the electroencephalogram recordings in WAG/RIJ rat model of absence epilepsy. Eur Rev Med Pharmacol Sci 17: 1149-1154, 2013.

2. Li XW, He H, Liu YF, Gao F, Wei D, Meng XD, Ma L and Jiang W: Effectiveness and safety assessment of lamotrigine monotherapy for treatment of epilepsy. Eur Rev Med Pharmacol Sci 16: 1409-1413, 2012.

3. Pal A, Nayak S, Sahu PK and Swain T: Piperine protects epilepsy associated depression: A study on role of monoamines. Eur Rev Med Pharmacol Sci 15: 1288-1295, 2011.

4. Brodie MJ: Road to refractory epilepsy: The Glasgow story. Epilepsia 54 (Suppl 2): 5-8, 2013.

5. Berg AT, Vickrey BG, Testa FM, Levy SR, Shinnar S, DiMario F and Smith S: How long does it take for epilepsy to become intractable? A prospective investigation. Ann Neurol 60: 73-79, 2006.

6. Zhen H,Zhen H and Dong Z: Definition of drug-resistant epilepsy: Unified proposal forwarded by the project team of International Union of Antiepileptic Treatment Strategy Committee. Chin J Neurol 43: 487-492, 2010.

7. Prasad AN and Hoffmann GF: Early onset epilepsy and inherited metabolic disorders: Diagnosis and management. Can J Neurol Sci 37: 350-358, 2010.

8. Di Iorio G, Matarazzo I, Di Tizio L and Martinotti G: Treatment-resistant insomnia treated with pregabalin. Eur Rey Med Pharmacol Sci 17: 1552-1554, 2013.

9. Wang YL, Xue F, Liu LZ and He ZH: Pathway analysis detect potential mechanism for familial combined hyperlipidemia. Eur Rev Med Pharmacol Sci 17: 1909-1915, 2013.

10. Krysiak R and Okopie B: Chronic adrenal failure and hypergonadotropic hypogonadism in a patient with abetalipoproteinemia. Eur Rev Med Pharmacol Sci 16 (Suppl 4): 95-97, 2012.

11. Vitiello G, De Clemente V, Della Casa R, Romano A, Rosa M, Pascarella A, Riccitelli M, Parenti G and Del Giudice E: Epilepsy in inherited metabolic disorders: A pediatric series. Minerva Pediatr 64: 513-520, 2012.

12. Xiaomei W, Cunming J and Yingbo Q: Screening diagnosis and treatment progress of inherited metabolic diseases on new born children. Inter J Gen 35: 21-24, 2012.

13. Kaspar H,Dettmer K, Chan Q, Daniels S, Nimkar S, Daviglus ML, Stamler J, Elliott P and Oefner PJ: Urinary amino acid analysis: a comparison of iTRAQ-LC-MS/MS, GC/MS, and amino acid analyzer. J Chromatogr B Analyt Technol Biomed Life Sci 877: 1838-1846, 2009.

14. Padda RS, Gkouvatsos K, Guido M, Mui J, Vali H and Pantopoulos K: A high-fat diet modulates iron metabolism but does not promote liver fibrosis in hemochromatotic $\mathrm{Hjv}^{-/}$mice mice. Am J Physiol Gastrointest Liver Physiol 308: G251-G261, 2015.
15. Nakagawa K, Kawana S, Hasegawa Y and Yamaguchi S: Simplified method for the chemical diagnosis of organic aciduria using GC/MS. J Chromatogr B Analyt Technol Biomed Life Sci 878: 942-948, 2010.

16. Campos HD: Tandem mass spectrometry as screening for inborn errors of metabolism. Rev Med Chil 139: 1356-1364, 2011 (In Spanish).

17. Yan L, Na Y and Chen F: Preliminary analysis on 552 children with inherited metabolic disorders through the screening of tandem mass spectrometry. Chin J Contemp Pediatr 13: 296-299, 2011 (In Chinese)

18. Ting A and Li L: An analysis of the screening status of inherited metabolic disorders on high-risk infants and young children in Xi'an in 2010. Chin J Child Care 20: 202-204, 2012.

19. López-Vicario C, Rius B, Alcaraz-Quiles J, García-Alonso V, Lopategi $\mathrm{A}$, Titos $\mathrm{E}$ and Clària $\mathrm{J}$ : Pro-resolving mediators produced from EPA and DHA: Overview of the pathways involved and their mechanisms in metabolic syndrome and related liver diseases. Eur J Pharmacol: May 15, 2015 (Epub ahead of print).

20. Taibi-Djennah Z and Laraba-Djebari F: Effect of cytokine antibodies in the immunomodulation of inflammatory response and metabolic disorders induced by scorpion venom. Int Immunopharmacol 27: 122-129, 2015.

21. Eisenstein A, Patterson S and Ravid K: The many faces of the A2b adenosine receptor in cardiovascular and metabolic diseases. J Cell Physiol 230: 2891-2897, 2015.

22. Miao D and Zhang L: Leptin modulates the expression of catabolic genes in rat nucleus pulposus cells through the mitogen-activated protein kinase and Janus kinase 2/signal transducer and activator of transcription 3 pathways. Mol Med Rep 12: 1761-1768, 2015.

23. Johnson CW and Beckham GT: Aromatic catabolic pathway selection for optimal production of pyruvate and lactate from lignin. Metab Eng 28: 240-247, 2015.

24. Neis EP, Dejong CH and Rensen SS: The role of microbial amino acid metabolism in host metabolism. Nutrients 7: 2930-2946, 2015.

25. Prystupa A, Szpetnar M, Boguszewska-Czubara A Grzybowski A, Sak J and Załuska W: Activity of MMP1 and MMP13 and amino acid metabolism in patients with alcoholic liver cirrhosis. Med Sci Monit 21: 1008-1014, 2015.

26. Enomoto H, Aizawa N, Nakamura H, Takata R, Sakai Y, Iwata Y, Tanaka H, Ikeda N, Aoki T, Hasegawa K, et al: A new metabolism-related index correlates with the degree of liver fibrosis in hepatitis $C$ virus-positive patients. Gastroenterol Res Pract 2015: 926169, 2015.

27. Wang X, Wei H, Cao J, Li Z and He P: Metabolomics analysis of muscle from piglets fed low protein diets supplemented with branched chain amino acids using HPLC-high-resolution MS. Electrophoresis 36: 2250-2258, 2015. 